\title{
Neuro-Neutrophilic Disease: Neuro-Behçet Disease and Neuro-Sweet Disease
}

\author{
Kinya Hisanaga \\ Key words: neuro-sweet disease, neuro-behçet disease, encephalitis, meningitis, HLA, neutrophil
}

(DOI: 10.2169/internalmedicine.46.0175)

"Neuro-Sweet disease (NSD)" was proposed as a distinct entity of encephalomeningitis in 1999 (1). Sweet disease has been considered as a multisystem inflammatory disorder characterized by painful erythematous plaques, and has been classified as a dermatologic disease. The plaques respond to systemic corticosteroids, and heal without scarring. It has been disclosed that aseptic neutrophilic inflammation occurs in the skin and in various organs including eyes, lungs, liver, kidneys, gastrointestinal tract, bone marrow, and muscle in Sweet disease. Encephalomeningitis also occurs in the disease, and was named NSD after the related inflammatory disorder neuro-Behçet disease (NBD).

Clinical manifestations and criteria for diagnosis of NSD were reported in 2005 (2). The encephalitis may occur in any CNS region, resulting in various central symptoms including headache and consciousness disturbance. Fever of 38 to $40^{\circ} \mathrm{C}$ is typically observed. Abnormal signal intensities on MRI are demonstrated in various CNS regions, and CSF studies have revealed mild increases in the protein concentration and mild to moderate pleocytosis. Increases in neutrophilis, erythrocyte sedimentation rate, and C-reactive protein in the peripheral blood are also demonstrated. Systemic corticosteroids, but not antibiotics, are usually very effective, although spontaneous remission may occur. The encephalomeningitis usually remits without apparent sequelae. Recurrence is not infrequent, and preventive therapy has not been established, although indomethacin, colchicine, and potassium iodide, in addition to corticosteroids, have been tried. The differences between NSD and NBD are as follows: 1) both sexes are almost evenly affected in NSD, while men are affected approximately 3.4 times as frequently as women with NBD; 2) people aged 30 to 70 are affected in NSD, whereas people aged 20 to 40 are preferentially affected in NBD; 3) any region of the CNS can be involved without site predilection, resulting in a variety of neurologic symptoms in NSD, while basal ganglia and brainstem are preferentially affected in NBD; 4) some patients with NSD demonstrate ocular signs including epis- cleritis and conjunctivitis, whereas uveitis is common in Behçet disease; 5) there is a strong human leukocyte antigen (HLA)-Cw1 and B54 association in NSD, while there is a high frequency of HLA-B51 in NBD.

Dermal infiltration with mature neutrophils in the absence of vasculitis in Sweet disease is different from dermal symptoms with vasculitis in Behçet disease; therefore, skin biopsies are necessary to diagnose "probable" NSD in the criteria. On the other hand, some mucocutaneous symptoms and signs without vasculitis including oral aphthae, benign genital ulcers, and pathergy reaction are common in both diseases.

The differential diagnosis of a few cases is not easy because the clinical features of both diseases are present. Neutrophils are considered to play a central role in the pathogenesis of both Sweet disease and Behçet disease, but the clinical phenotype may differ because of the differences in genetic background including HLA type. In this journal, Tsunemi et al (3) reported an interesting case which fulfilled the criteria for the diagnosis of Behçet disease as well as "possible" NSD, although the diagnosis of NSD was not confirmed by skin biopsy. The question of the proper diagnosis of such cases in the border zone remains. Pathological findings in the CNS have been demonstrated to be different between NBD and NSD. In NBD, vasculitis is also seen in the CNS as well as in dermal lesions, which likely results in the poorer prognosis associated with the CNS lesions. On the other hand, vasculitis may be absent in the CNS lesions as well as in the typical eruptions in $\operatorname{NSD}(4,5)$. Therefore, the presence or absence of vasculitis in the skin lesions appears to be important for the differentiation between these related diseases. However, dermal involvement does not necessarily precede neurologic involvement. Skin biopsy can not be always performed timely so that it may fail to demonstrate the typical pathological findings. It may not be appropriate to differentiate NSD from NBD by only the neurologic symptoms and signs, findings in brain MRI and CSF studies, and laboratory data including HLA type, even if the 


\section{Neuro-neutrophilic disease}

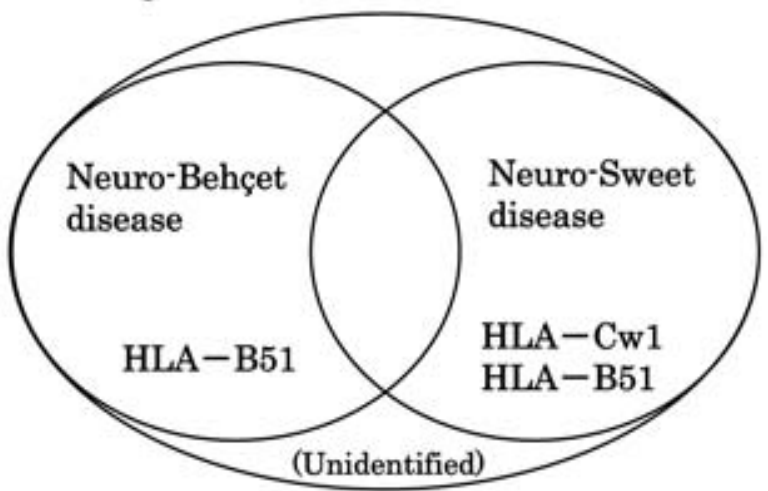

Figure. Neuro-neutrophilic disease patients demonstrate benign, steroid-responsive encephalomeningitis and are HLA-Cw1 or B54 positive. Therefore, a tentative diagnosis is necessary for the cases without confirmation by skin biopsy. Dermatologic diseases in which neutrophils play a core role in the pathogenesis are called "neutrophilic dermatosis". "Neutrophilic disease" is also used as a name for such diseases involving multiple organs. It may be reasonable to tentatively call NBD and / or NSD as "neuro-neutrophilic disease". It may be useful to classify NBD as a relatively malignant type of neuro-neutrophilic disease, and classify NSD as a benign type. The case of Tsunemi (5) can be tentatively classified as a border type. It will be important to carefully follow-up new dermatologic signs in the future in such cases.

\section{References}

1. Hisanaga K, Hosokawa M, Sato N, Mochizuki H, Itoyama $\mathrm{Y}$, Iwasaki Y. "Neuro-Sweet Disease" benign recurrent encephalitis with neutrophilic dermatosis. Arch Neurol 56: 1010-1013, 1999.

2. Hisanaga K, Iwasaki Y, Itoyama Y, Neuro-Sweet Disease Study Group. Neuro-Sweet disease: Clinical manifestations and criteria for diagnosis. Neurology 64: 1756-1761, 2005.

3. Tsunemi T, Sakai Y, Tsunoda K, et al. Neuro-Behçet's/neuro-
Sweet's disease presents simultaneously with severe tonsillitis, and features mimicking bacterial meningitis with skin lesions. Intern Med 45: 1315-1317, 2006.

4. Takiyama Y, Ogino M, Ogino Y, et al. Neuroimmunology 13: 131, 2005 (in Japanese).

5. Kokubo Y, Kuzuhara S. Neuropathological aspects of neuro-Sweet disease. Neurological Medicine 64: 141-147, 2006 (in Japanese).

(C) 2007 The Japanese Society of Internal Medicine http://www.naika.or.jp/imindex.html 